\title{
Genetic analysis of Thai cattle reveals a Southeast Asian indicine ancestry
}

Pongsakorn Wangkumhang, Alisa Wilantho, Philip J Shaw, Laurence Flori, Katayoun Moazami-Goudarzi, Mathieu Gautier, Monchai Duangjinda, Anunchai Assawamakin, Sissades Tongsima

Cattle commonly raised in Thailand have characteristics of Bos indicus (zebu). We do not know when or how cattle domestication in Thailand occurred, and so questions remain of their origins and relationships to other breeds. We obtained genome-wide SNP genotypic data of 28 bovine individuals sampled from four regions: North (Kho-Khaolampoon), Northeast (Kho-Isaan), Central (Kho-Lan) and South (Kho-Chon) Thailand. These regional varieties have distinctive traits suggestive of breed-like genetic variations. From these data, we confirmed that all four Thai varieties are Bos indicus and that they are distinct from other indicine breeds. Among these Thai cattle, a distinctive ancestry pattern is apparent, which is the purest within Kho-Chon individuals. This ancestral component is only present outside of Thailand among other indicine breeds in Southeast Asia. From this pattern, we conclude that a unique Bos indicus ancestor originated in Southeast Asia, and native Kho-Chon Thai cattle retain the signal of this ancestry with limited admixture of other bovine ancestors. 


\section{Genetic analysis of Thai cattle reveals a Southeast Asian indicine ancestry}

3 ABSTRACT

4 Cattle commonly raised in Thailand have characteristics of Bos indicus (zebu). We do not know

5 when or how cattle domestication in Thailand occurred, and so questions remain of their origins

6 and relationships to other breeds. We obtained genome-wide SNP genotypic data of 28 bovine

7 individuals sampled from four regions: North (Kho-Khaolampoon), Northeast (Kho-Isaan),

8 Central (Kho-Lan) and South (Kho-Chon) Thailand. These regional varieties have distinctive

9 traits suggestive of breed-like genetic variations. From these data, we confirmed that all four

10 Thai varieties are Bos indicus and that they are distinct from other indicine breeds. Among these

11 Thai cattle, a distinctive ancestry pattern is apparent, which is the purest within Kho-Chon

12 individuals. This ancestral component is only present outside of Thailand among other indicine

13 breeds in Southeast Asia. From this pattern, we conclude that a unique Bos indicus ancestor

14 originated in Southeast Asia, and native Kho-Chon Thai cattle retain the signal of this ancestry

15 with limited admixture of other bovine ancestors.

17 Keywords: Thai bovine, Kho-Khaolampoon, Kho-Isaan, Kho-Lan, Kho-Chon, Population 18 genetics, ipPCA, Admixture, Population structure

21 Pongsakorn Wangkumhang, Genome Technology Research Unit, National Center for Genetic

22 Engineering and Biotechnology, Pathum Thani, Thailand

23 Alisa Wilantho, Genome Technology Research Unit, National Center for Genetic Engineering 24 and Biotechnology, Pathum Thani, Thailand

25 Philip James Shaw, Medical Molecular Biology Research Unit, National Center for Genetic 26 Engineering and Biotechnology, Pathum Thani, Thailand

27 Laurence Flori, INRA, UMR 1313 GABI, F78350 Jouy-en-Josas, France, CIRAD, UMR

28 INTERTRYP, F34398 Montpellier, France

29 Katayoun Moazami-Goudarzi, INRA, UMR 1313 GABI, F78350 Jouy-en-Josas, France

30 Mathieu Gautier, INRA, UMR CBGP (INRA/CIRAD/IRD/Supagro), F34988 Montferrier-sur-

31 Lez, France 
32 Monchai Duangjinda, Department of Animal Science, Faculty of Agriculture, Khon Kaen

33 University, Khon Kaen, Thailand

34 Anunchai Assawamakin, Department of Pharmacology, Faculty of Pharmacy, Mahidol

35 University, Bangkok, Thailand

36 corresponding author: Sissades Tongsima, Genome Technology Research Unit, National Center

37 for Genetic Engineering and Biotechnology, Pathum Thani, Thailand, Tel. +662 5646700, email:

38 sissades@biotec.or.th

\section{Introduction}

41 Following the completion of the bovine genome, genome-wide studies have been conducted to 42 catalog genetic variants, e.g. the bovine Hapmap project. Bovine genetic studies have been conducted extensively among European and taurine cattle (Beja-Pereira et al., 2006; Dadi, 2014;

44 Gautier et al., 2010; Gorbach et al., 2010; Lee et al., 2014; McTavish et al., 2013; Speller et al., 45 2013; Suh et al., 2014). However, little is known about Asian cattle, which are predominantly Bos indicus. Recently, a worldwide study of bovines including Asian Bos indicus breeds from India, Pakistan, China and Indonesia showed evidence of Bos javanicus ancestry that contributes to Asian bovine diversity (Decker et al., 2014). However, no one has yet investigated the ancestry of Thai cattle, and the domestication of bovine in Southeast Asia is poorly understood (Larson \& Fuller, 2014). Therefore, to address this question further study of Asian Bos indicus

51 breeds is needed.

Cattle native to Thailand (excluding recently introduced European breeds) have Bos indicus traits, including the distinctive dorsal hump. However, to our knowledge, there has been no formal genetic testing that these cattle are Bos indicus. Genetic variation of native Thai bovines has been studied using different marker types, i.e., STR (Jirajaroenrat et al., 2008a), mitochondrial DNA (Jirajaroenrat et al., 2008b; Kornphan et al., 2012; Sarataphan et al., 2013), and SNPs (Charoensook et al., 2011; Edea et al., 2013). However, none of these studies provide any information of the origins and ancestries of these cattle, since the markers used are insufficiently informative. The earliest evidence of domestic cattle in Thailand dates from 4000-

615000 B.C.E (MacHugh, 1996). There are four native breeds officially recognized by the 
63 Thailand origin, THR), Kho-Isaan (northeastern Thailand, THNE), Kho-Lan (central Thailand, 64 THC) and Kho-Chon (southern Thailand, THS) (Fig. 1). According to folklore, these breeds may

\section{6}

have originated from different regions of Thailand. To our knowledge, there is no historical evidence of any domestication event for these breeds, and so it is unclear if they can be distinguished as breeds in the accepted sense. A breed can be defined as a group of individuals with shared distinctive phenotypic traits and a genetic signature that distinguishes them from other breeds. The phenotypic traits of Thai cattle that are breed characteristics include coat color and body size. THNE coat colors vary from red, black, brown, white to yellow. They have the smallest body size of all Thai breeds. THC and THR have body sizes similar to THNE. The THR breed is considered to possess traits suitable for presentation in the Thai royal plough day ceremony (early May of every year), which may have been performed since the Sukhothai era $\left(13^{\text {th }}\right.$ Century CE). THS coat colors are light/dark brown and black. THS are mainly used as draught animals owing to their large body size relative to other Thai breeds. These Thai cattle are well adapted to the tropical environment, e.g., heat tolerance and resistance to ectoparasites (Akkahart, 2003; Charoensook et al., 2011; Kahi, 2004; Khamkwan et al., 2012a; Khamkwan et al., 2012b; Saithong et al., 2011). Notwithstanding these breed characteristics, it is unknown if these four Thai native breeds can be distinguished genetically as breeds, and what their possible origins could be in relation to other cattle.

In this work, we present a population genetics study of 28 individuals sampled from the four Thai native breeds. Genotyping data were obtained using the Illumina BovineSNP50K chip array platform. These data were analyzed together with 1,369 worldwide cattle from 88 breeds previously published (Decker et al., 2014).

\section{Materials and Methods}

\section{Ethical statement}

In this study, we obtained samples from two sources. Tissue samples were provided by Dr. Yanin Opaspattanakit, Maejo University, Thailand, which were obtained as part of an ongoing Government program, sponsored by the Thailand Research Fund (project code RDC492001) for improving Thai native cattle production (Department of Livestock Development. Available 
94 from: http://www.dld.go.th/th/images/stories/news/Strategy/55-59\%20strategy_beef.pdf). The

95 full list of researchers who contributed the samples in this program is listed in Table S1. 96

97 We also obtained blood samples specifically for this study. The studied animals were maintained 98 at the Khon Kaen University (KKU) beef farm, Thailand. This facility is owned by Khon Kaen 99 University for the purpose of Agricultural scientific research. Hence, no field permit is required 100 to study them. Five to ten milliliters of blood were taken from the jugular or tail vein. All efforts were made to minimize distress when taking blood samples. The protocols for recruiting animals and drawing blood were approved by the Animal Ethics Committee of Khon Kaen University under the permit number AEKKU 41/2557.

Data used

\section{- Thai bovine SNP data}

Tissue and blood samples were obtained from 28 individuals. DNA was extracted from tissues using $\mathrm{Gu}-\mathrm{HCl}$ (Pramanick et al., 1976). DNA was extracted from blood using a QIAamp DNA Blood Mini kit (QIAGEN, Hilden, Germany). Final DNA concentrations ranged from 43-125 $\mu \mathrm{g} / \mathrm{ml}$ (Table S2). Blood samples were obtained from THNE individuals, whereas tissue samples were taken from the rest (THR ( 8 individuals), THC (5 individuals) and THS (5 individuals)).

112 Genotyping was performed using the Illumina BovineSNP50 chip at the INRA Labogena

113 platform (Jouy-en-Josas, France) using standard procedures (http://www.illumina.com). The raw genotypic data are available in Dataset S3. The same data quality control process as described in

115 Gautier et al. (Gautier et al., 2010) was used, which left 44,706 markers passing the quality 116 control. These markers were used in all subsequent analyses. To check whether closely related 117 individuals were sampled, we performed identity-by-decent (IBD) test as recommended by

118 (Porto-Neto et al., 2013). IBD values were calculated in pairwise combinations of individuals 119 using PLINK software (Purcell et al., 2007).

Worldwide bovine SNP data

122 The dataset containing 1,365 individuals from 87 worldwide bovine breeds was downloaded 123 from http://dx.doi.org/10.5061/dryad.th092, published in (Decker et al., 2014). This dataset 124 includes data from other studies (Gautier et al. (Gautier et al., 2009) and Gautier et al. (Gautier et 
125 al., 2010)). We also included four individuals of Bison bison (OBB) described in (Gautier et al., 126 2010). Full information of sampled individuals is shown in Table S4.

\section{Fst and informative marker selection genetic distance among bovine breeds}

129 In this analysis, the Thai individuals were assumed to belong to the same group of Thai cattle.

130 All other cattle were grouped according to breed labels. Genetic distance between breeds

131 according to groups of individuals was calculated using the pairwise Weir \& Cockerham Fst

132 equation (Weir, 1984).

133

\section{Phylogenetic analysis}

135 Bootstrapped phylogenetic trees were constructed using Phylip (Felsenstein, 2005). A pairwise

136 distance matrix among breeds was constructed using Nei's genetic distance. All Thai cattle were

137 put into one group. 100 bootstraps were performed with random $10 \%$ marker resampling. The

138 consensus tree was constructed from the bootstrapped data with bootstrapping confidence values

139 assigned to each node using FigTree version 1.4.2 (graphical viewer accompanying BEAST;

140 Drummond \& Rambaut, 2007).

\section{Unsupervised population clustering}

143 Iterative Pruning Principal Component Analysis (ipPCA; Limpiti et al., 2011) was used to cluster

144 individuals into subpopulation groups. This unsupervised clustering algorithm uses Principal

145 Component Analysis (PCA). The EigenDev heuristic is employed on the Eigenvalues from PCA

146 to detect whether substructure exists. If this condition is met (EigenDev $>0.21)$, the individuals

147 are separated into two groups using high-dimensional fuzzy c-means clustering. The procedure is

148 iterated until no substructure exists among the individuals, which are defined as subpopulations.

149 The ipPCA algorithm can be used to assign individuals to groups without assumptions of

150 ancestry from population, i.e., breed labels. The theoretical basis for why the iterative clustering

151 approach used in ipPCA can provide greater accuracy is described in (Limpiti et al., 2011).

152

153 We extended the ipPCA analysis framework by testing the robustness of subpopulation

154 assignments. In this extra procedure, $10 \%$ of the markers were randomly sampled (with

155 replacement) using a random generator library implemented in MATLAB version R2009b, and 
156 the resampled genotypic data were used as input for ipPCA. 1000 bootstrap resampled datasets

157 were generated and the consensus subpopulation assignment map was constructed. The bootstrap

158 confidence values were calculated from the percentage of individuals assigned in all bootstrap

159 datasets (Table S5).

160

161

ADMIXTURE analysis

162 The ADMIXTURE software (Alexander et al., 2009) was used for inferring ancestry ratios. This

163 program uses maximum likelihood modeling to estimate ancestry, which is much faster than

164 Bayesian modeled ancestry as implemented in STRUCTURE (Pritchard et al., 2000). The

165 number of $K$-ancestors was varied from $K=2$ to $K=100$ (whole dataset), and $K=2$ to $K=30$

166 (indicine only). Ten cross-validations were performed to estimate cross-validation error for

167 determining the suitable number of $K$-ancestors for interpretation. The cross-validation plots are

168 shown in Fig. S6. Individual ancestry patterns were generated using CLUMPP (Jakobsson \&

169 Rosenberg, 2007) from the ADMIXTURE outputs. Individuals in Fig. 4A were sorted according

170 to the subpopulation assignments made by ipPCA (see Dataset S7 for the full result of the

171 ADMIXTURE analysis). To test whether cryptic relatedness among Thai individuals could cause

172 artifactual groupings of inferred ancestry in ADMIXTURE analysis, ADMIXTURE was

173 performed with 268 indicine individuals plus a Thai individual. This process was repeated for all

17428 Thai individuals. The Q value of the Southeast Asian ancestral component, which is the major

175 ancestral component value among PES and ACE Indonesian breeds, was extracted from the Thai

176 individual in each analysis.

177

178 Results and Discussion

179 We used four different approaches to ascertain the population structure and genetic identities of

180 Thai native cattle. First, pairwise comparisons of all breeds were performed using Fst analysis. In

181 this analysis, the Thai individuals were placed into one group as we have no prior evidence that

182 the four breeds are actually genetically distinct groups. The Thai cattle are clearly identified as

183 Bos indicus (Fig. 2). Next, to discern in more detail the relationship of Thai to other indicine

184 cattle, NJ tree analysis was performed. Thai cattle occupy a position in the tree among other

185 Southeast Asian indicine breeds (PES, ACE, BRE, MAD, and BALI) with 100\% bootstrap

186 support (Fig. 3). In particular, PES and ACE breeds are on a node closer to Thai than BRE, 
187 MAD and BALI. The BRE and MAD breeds are Bos indicus with considerable Bos javanicus 188 ancestry (represented by BALI) (Decker et al. 2014). Hence, Thai cattle are less likely to have 189 admixture of Bos javanicus. Although the Thai cattle occupy a distinct position in the tree with 190 their Southeast Asian neighbors, there is ambiguity in the relationship to other Asian indicine 191 breeds (RSIN, DHA, KAN, and SAHW) as shown by the nodes with weaker support $(<80 \%)$.

192

193 We tested the hypothesis that cattle breeds define genetically homogenous subpopulations of 194 individuals using a clustering approach that is blind to the breed information. This approach, implemented using the ipPCA algorithm, identified 96 genetically distinct subpopulations (Table

196 S5). The majority of these subpopulations have strong bootstrap supports in ipPCA, which in the main comprise individuals with the same breed label. However, a direct correspondence between breeds and genetically homogenous subpopulations does not always exist. For example, SP28, SP29 and SP30 comprise individuals from the same NORM breed (see Fig. S8). Moreover, some confidently assigned ( $\geq 95 \%$ bootstrap) subpopulations comprise individuals with different breed labels, including SP4-9, SP11-13, SP15, SP18-33, SP35-40, SP42-60, SP62-64, SP68-76, SP7981, SP89, SP90, SP93, SP95, and SP96. Genetically distinct subpopulations among individuals of the same breed may arise from drift, particularly for breeds that were established a long time ago. Conversely, breeds with recent common ancestry may not be distinguished as genetically distinct subpopulations with the genetic markers available. Although ipPCA assignments were robust in the main, some subpopulations had weak bootstrap support $(\leq 80 \%)$ indicating that assignments of these individuals are inaccurate. The inaccuracy in cluster assignment by ipPCA for these subpopulations reflects the weak bootstrap support of the same individuals in the NJ tree, e.g., SP85-87 contain individuals from South Asian breeds (THA, RSIN, HAR, KAN, $\mathrm{CHO}, \mathrm{DAJ}, \mathrm{LOH}$, and $\mathrm{ACH})$. Cluster assignment of Southeast Asian breeds from Indonesia is

211 also somewhat inaccurate in that only ACE (SP91) is likely to be a genetically distinct group 212 (88\% bootstrap support).

214 Thai cattle were assigned to SP88, SP89, and SP90. THS and THNE individuals comprise SP89 215 and SP90 respectively, which were assigned with strong bootstrap support ( $\geq 95 \%)$. In contrast, 216 SP88 comprises individuals from three Thai breeds, THC, THNE, and THR with weaker 217 bootstrap support. Therefore, three distinct subpopulations can be resolved among the Thai 
218 cattle. It is interesting to note that THR, which is revered as a Thai cultural icon, is genetically

219 similar to other Thai cattle (THNE and THC). The only Thai breed that appears as genetically

220 distinct group is THS.

221

222 Ancestry was determined by ADMIXTURE analysis. At $K=2$ modeled ancestors, the major

223 ancestral component of taurine cattle is clearly distinct from the major component of indicine

224 cattle (Fig. 4A). Taurine cattle were assigned predominantly to SP12 to SP53 by ipPCA, and

225 they share a major ancestral component (red). In contrast, ipPCA subpopulations SP64 to SP94

226 comprise indicine individuals that share the other major ancestral component (yellow).

227 Individuals of hybrid breeds, e.g., Beefmaster (BEFM) assigned to SP54, exhibit both ancestral

228 components. According to the cross-validation calculation in ADMIXTURE, $K=55$ has the

229 lowest cross-validation error and thus it is a suitable number of ancestral components for the

230 entire population dataset (Fig. S6A). Patterns specific to each subpopulation are apparent at

$231 K=55$, in particular the groups with assigned European taurine individuals. Within the

232 subpopulations containing mostly indicine individuals, the individuals within SP88 to SP94 share

233 a unique major ancestral component.

235 Having shown that Thai cattle are indicine, we investigated ancestry patterns within the indicine

236 cattle in greater detail by performing ADMIXTURE on the indicine cattle only. According to the

237 cross-validation calculation in ADMIXTURE, $K=11$ is a suitable number of ancestral

238 components for the indicine sub-dataset (Fig. S6B). The Southeast Asian ACE, PES and Thai

239 cattle shared a major ancestral component (dark blue) distinct from other indicine, including

240 MAD and BRE Southeast Asian cattle (Fig. 4B). This result is in agreement with the NJ tree

241 shown in Fig. 2, in which MAD and BRE are distinct from other Southeast Asian cattle because

242 of Bos javanicus admixture. Among the Thai cattle, the THS have the least admixture of other

243 components. The distinctiveness of the THS individuals could be the result of cryptic

244 relatedness, since IBD scores are markedly higher than 0.1 (Table S9). Sampling of relatives is

245 not congruent with the ADMIXTURE model assumption that individuals are independent

246 (Alexander et al. 2009). To reduce the possible effect of cryptic relatedness among Thai

247 individuals, we performed another set of ADMIXTURE analyses in which indicine cattle were

248 analyzed with a single Thai individual, and repeated for all 28 Thai cattle. In these analyses, the 
optimal $K$ inferred from cross-validation error was $K=8$ for all 28 experiments of ADMIXTURE

250 (Fig. S5C). From the NJ analysis and earlier ADMIXTURE analyses, we used the assumption

251 that the major ancestral component of ACE and PES individuals was shared with the Thai cattle.

252 The variation of this Southeast Asian ancestry among the Thai individuals in shown in Fig. 4C.

253 The THS individuals have a markedly greater proportion (0.65-0.77) of Southeast Asian ancestry

254 from the others (THC, THR, and THNE), such that THS can be considered as a genetically

255 distinct breed. It should be noted that the sampling of THS and other Thai cattle is rather low and

256 could be biased. Therefore, the conclusion about the uniqueness of these cattle must take this

257 caveat into account. The uniqueness of the Southeast Asian cattle is perhaps somewhat surprising 258 given the known ascertainment bias of SNP markers toward taurine breeds (Matukumalli et al., 259 2009; McTavish et al., 2013). This is obvious from our admixture analysis in which the Bison

260 bison and Bos javanicus are indistinguishable from indicine breeds at low numbers of $K$

261 ancestors ( $K=2$ to 5 ; Dataset S7).

262

263 In conclusion, Thai cattle are indicine that are most closely related to other Southeast Asian

264 breeds. The Kho-Chon variety (THS) appears to be a distinct breed with minimal admixture. All

265 other Thai and Southeast Asian cattle show evidence of admixture. However, this claim must be

266 tempered against the limitation of the data available, in particular the marker platform and

267 sampling of individuals. Moreover, the weak bootstrap support from NJ tree and ipPCA

268 clustering among South Asian indicine breeds further points to lack of power to differentiate

269 indicine breeds. Future studies into bovine genetic diversity should include whole genome

270 sequencing to discover new variants among indicine breeds. Kho-Chon Thai cattle are of

271 particular interest for further genomic study into the Southeast Asian Bos indicus ancestor.

272

\section{Acknowledgements}

274 We thank Dr. Yanin Opaspattanakit, Maejo University for providing tissue samples. We also

275 acknowledge Ms. Thongsa Buasook and Dr. Thevin Vongpralab, Department of Animal Science,

276 Faculty of Agriculture, KhonKaen University for their assistance in collecting blood samples.

277 We acknowledge the Thai department of Livestock Development, Ministry of Agriculture and

278 Cooperatives for providing images of the Thai native cattle. The geographical map used in Fig. 1 
279 is courtesy of the U.S. Geological Survey. Finally, we thank the Editor and reviewers for their 280 suggestions to improve the manuscript.

281

\section{References}

283 Department of Livestock Development. Available from: http://www.dld.go.th/th/images/stories/news/Strategy/55-59\%20strategy_beef.pdf. (accessed 15 June 2015).

Department of Livestock Development.Statistic report of Thai cattle. Available from: http://ict.dld.go.th/th2/index.php/en/report-menu-en/13-statistic. (accessed 3 June 2015).

Akkahart K. 2003. Line clustering and estimation of genetic parameters for growth traits and body measurements in Thai native cattle. Thesis, Khon Kaen University, KhonKaen, Thailand.

Alexander DH, Novembre J, and Lange K. 2009. Fast model-based estimation of ancestry in unrelated individuals. Genome Research 19:1655-1664.

Beja-Pereira A, Caramelli D, Lalueza-Fox C, Vernesi C, Ferrand N, Casoli A, Goyache F, Royo LJ, Conti S, Lari M, Martini A, Ouragh L, Magid A, Atash A, Zsolnai A, Boscato P, Triantaphylidis C, Ploumi K, Sineo L, Mallegni F, Taberlet P, Erhardt G,Sampietro L, Bertranpetit J, Barbujani G, Luikart G, and Bertorelle G. 2006. The origin of European cattle: evidence from modern and ancient DNA. PNAS 103:8113-8118.

Charoensook R, Gatphayak K, Sharifi AR, Chaisongkram C, Brenig B, and Knorr C. 2011. Polymorphisms in the bovine HSP90AB1 gene are associated with heat tolerance in Thai indigenous cattle. Tropical Animal Health and Production 44:921-928.

Dadi H, Lee SH, Lee SS, Park C, Kim KS. 2014. Inter- and intra-population genetic divergence of East Asian cattle populations: focusing on Korean cattle. Genes\& Genomics 36.

Decker JE, McKay SD, Rolf MM, Kim J, Molina Alcala A, Sonstegard TS, Hanotte O, Gotherstrom A, Seabury CM, Praharani L, Babar ME, Correia de Almeida Regitano L, Yildiz MA, Heaton MP, Liu WS, Lei CZ, Reecy JM, Saif-Ur-Rehman M, Schnabel RD, and Taylor JF. 2014. Worldwide patterns of ancestry, divergence, and admixture in domesticated cattle. PLoS Genetics 10:e1004254.

Drummond AJ, and Rambaut A. 2007. BEAST: Bayesian evolutionary analysis by sampling trees. BMC Evolutionary Biology 7:214. 
310 Edea Z, Dadi H, Kim SW, Dessie T, Lee T, Kim H, Kim JJ, and Kim KS. 2013. Genetic

311

312

313

314

315

316

317

318

319

320

321

322

323

324

325

326

327

328

329

330

331

332

333

334

335

336

337

338

339

340 diversity, population structure and relationships in indigenous cattle populations of Ethiopia and Korean Hanwoo breeds using SNP markers. Frontiers in genetics 4:35. Felsenstein J. 2005. PHYLIP (PhylogenyInference Package) version 3.6. Distributed by the author Department of Genome Sciences, University of Washington, Seattle.

Gautier M, Flori L, Riebler A, Jaffrezic F, Laloe D, Gut I, Moazami-Goudarzi K, and Foulley JL. 2009. A whole genome Bayesian scan for adaptive genetic divergence in West African cattle. BMC Genomics 10:550.

Gautier M, Laloe D, and Moazami-Goudarzi K. 2010. Insights into the genetic history of French cattle from dense SNP data on 47 worldwide breeds. PLoS One 5.

Gorbach DM, Makgahlela ML, Reecy JM, Kemp SJ, Baltenweck I, Ouma R, Mwai O, Marshall K, Murdoch B, Moore S, and Rothschild MF. 2010. Use of SNP genotyping to determine pedigree and breed composition of dairy cattle in Kenya. Journal of Animal Breeding and Genetics 127:348-351.

Jakobsson M, and Rosenberg NA. 2007. CLUMPP: a cluster matching and permutation program for dealing with label switching and multimodality in analysis of population structure. Bioinformatics 23:1801-1806.

Jirajaroenrat K, Boonwong P, Tuntivisoottikul K. 2008a. Genetics diversity of Thai Indigenous Beef Cattle Lines Using Microsatellites. In The 13th Animal Science Congress of the Asian-Australasian Association of Animal Production Societies Hanoi: Vietnam September 22-26.

Jirajaroenrat K, Satitmanwiwat S, Tuntivisoottikul K. 2008b. Genetic Diversity of Central Thailand Beef Cattle Revealed by Mitochondrial DNA Data. In The 13th Animal Science Congress of the Asian-Australasian Association of Animal Production Societies Hanoi: Vietnam September 22-26.

Kahi AKGH. 2004. Indigenous Thai Beef Cattle Breeding Scheme Incorporating Indirect Measures of Adaptation : Sensitivity to Changes in Heritabilities of and Genetic Correlations between Adaptation Traits. Asian-Australasian Journal of Animal Sciences 17:1039-1046.

Khamkwan D, Laepaijit Y, Vitoonpong E, and Intaratham W. 2012a. Effect of Inbreeding on growth traits in Esarn indigenous cattle. BAHGI e-journal 2:15-27. (article in Thai). 
341 Khamkwan D, Laepaijit Y, Vitoonpong E, and Intaratham W. 2012b. Estimated breeding value

342

343

344

345

346

347

348

349

350

351

352

353

354

355

356

357

358

359

360

361

362

363

364

365

366

367

368

369

370 and genetic trend of growth traits in Southern indigenous cattle line from multivariate analysis. BAHGI e-journal 2:1-14. (article in Thai).

Kornphan P, Akaboot P, Duangjinda M, Phasuk Y, Pakdee P. 2012. Genetic variation and cluster analysis of Thai native cattle using mitochondrial DNA (mtDNA). Khon Kaen Agriculture Journal:362-366.

Larson G, and Fuller D. 2014. The Evolution of Animal Domestication. The Annual Review of Ecology, Evolution, and Systematics 45:115-136.

Lee SH, Park B, Sharma A, Dang CG, Lee SS, Choi TJ, Choy YH, Kim HC, Jeon KJ, Kim SD, Yeon SH, Park SB, and Kang HS. 2014. Hanwoo cattle: origin, domestication, breeding strategies and genomic selection. Journal of Animal Science and Technology 56:8.

Limpiti T, Intarapanich A, Assawamakin A, Shaw PJ, Wangkumhang P, Piriyapongsa J, Ngamphiw C, and Tongsima S. 2011. Study of large and highly stratified population datasets by combining iterative pruning principal component analysis and structure. $B M C$ Bioinformatics 12:255.

Limpiti T, Intarapanich A, Assawamakin A, Wangkumhang P, Tongsima S 2011. Iterative PCA for population structure analysis. IEEE International Conference on Acoustics, Speech and Signal Processing (ICASSP), Prague Congress Centre, Prague, Czech Republic:597600.

MacHugh DE. 1996. Molecular biogeography and genetic structure of domesticated cattle. Thesis, Smurfit Institute of Genetics, Trinity College, Dublin, Ireland available: wwwgentcdie/molpopgen/thesesphp (accessed 3 June 2015).

Matukumalli LK, Lawley CT, Schnabel RD, Taylor JF, Allan MF, Heaton MP, O'Connell J, Moore SS, Smith TP, Sonstegard TS, and Van Tassell CP. 2009. Development and characterization of a high density SNP genotyping assay for cattle. PLoS One 4:e5350.

McTavish EJ, Decker JE, Schnabel RD, Taylor JF, and Hillis DM. 2013. New World cattle show ancestry from multiple independent domestication events. PNAS 110:E1398-1406.

Porto-Neto LR, Sonstegard TS, Liu GE, Bickhart DM, Da Silva MV, Machado MA, Utsunomiya YT, Garcia JF, Gondro C, and Van Tassell CP. 2013. Genomic divergence of zebu and taurine cattle identified through high-density SNP genotyping. BMC Genomics 14:876. 
371 Pramanick D, Forstova J, and Pivec L. 1976. 4 M guanidine hydrochloride applied to the

372

373

374

375

376

377

378

379

380

381

382

383

384

385

386

387

388

389

390

391

392

393

394

395

396

397

398

399

400

401 isolation of DNA from different sources. FEBS Letters 62:81-84.

Pritchard JK, Stephens M, and Donnelly P. 2000. Inference of population structure using multilocus genotype data. Genetics 155:945-959.

Purcell S, Neale B, Todd-Brown K, Thomas L, Ferreira MAR, Bender D, Maller J, Sklar P, de Bakker PIW, Daly MJ \& Sham PC. 2007. PLINK: a toolset for whole-genome association and population-based linkage analysis. American Journal of Human Genetics, 81.

Saithong S, Chatchawan T, Boonyanuwat K. 2011. Thai Indigenous Cattle Production Provide a Sustainable Alternative for the Benefit of Smallscale Farmers, Healthy Food, and the Environment. Journal of the Bureau of Animal Husbandry:21-26.

Sarataphan N, Chuensuk S, Apimeteetumrong M, Yiengvisawakul W, Teepatimakorn S. 2013. Genetic diversity of maternal lineages in Thai-native cattle by mitochondrial DNA analysis. Thai Journal of Agricultural Science 44:175-178.

Speller CF, Burley DV, Woodward RP, and Yang DY. 2013. Ancient mtDNAanalysis of early 16(th) century Caribbean cattle provides insight into founding populations of New World creole cattle breeds. PLoS One 8:e69584.

Suh S, Kim YS, Cho CY, Byun MJ, Choi SB, Ko YG, Lee CW, Jung KS, Bae KH, and Kim JH. 2014. Assessment of Genetic Diversity, Relationships and Structure among Korean Native Cattle Breeds Using Microsatellite Markers. Asian-Australasian Journal of Animal Sciences 27:1548-1553.

Weir B, Cockerham C. 1984. Estimating F-Statistics for the Analysis of Population Structure. Evolution 38:1358-1370.

\section{List of Figures}

Figure 1 Images of four native Thai cattle varieties. A) Kho-Khaolumpoon (THR), B) Kho-Isaan (THNE), C) Kho-Lan (THC), and D) Kho-Chon (THS) (Image courtesy of Department of Livestock Development). The assumed geographical origins of the varieties and other Asian breeds, namely Ongole Grade (ONG), Aceh (ACE), Pesisir (PES), Brebes (BRE), Bali (BALI), and Madura (MAD), are shown. 
402 Figure 2 Heat map of pair-wise Fst. Each cell represents a group of individuals of the same

403 breed; the heat map colors reflect Weir \& Cockerham Fst values as shown by the color scale bar 404 on the right. The data from 1397 animals genotyped for 44,706 markers were used to calculate 405 Fst values. The gray cell represents Bison bison (OBB) and the magenta cell represents Bos 406 javanicus (BALI), which are non-cattle reference animals. The Thai native cattle (28 individuals 407 from THS, THR, THC, and THNE) are indicated by a green bar. Indicus, Taurine, 408 Indicus/Taurine hybrid breeds are grouped arbitrarily, as indicated by brackets. 409

410 Figure 3 Unrooted Neighbor-Joining phylogenetic tree of cattle breeds. A pair-wise distance 411 matrix between breeds was calculated based on minor allele frequency (MAF). The data from 4121397 animals genotyped for 44,706 markers were used to calculate MAF values. The consensus 413 tree is shown from 100 bootstrap replicates. The bootstrap confidence intervals $(\leq 80 \%$, red 414 circle; $80-95 \%$, yellow square; $\geq 95 \%$, green star) are indicated at each node. Taurine breeds are 415 highlighted in blue, Hybrid breeds in green and Indicine breeds in yellow. Southeast Asian cattle 416 are outlined in black. The Thai cattle (TH, 28 individuals from THS, THR, THC, and THNE) are 417 highlighted in red. The non-cattle out-groups Bison bison (OBB) and Bos javanicus (BALI) are 418 highlighted in pink.

419

420 Figure 4 Combined result of ipPCA and ADMIXTURE analysis. The data from 1397 animals 421 genotyped for 44,706 markers were used in ipPCA to assign individuals to subpopulation groups 422 (SP1-SP96). Each SP contains individuals with no significant substructures detected by ipPCA. 423 The SP assignment is independent of the breed label information. To assess the confidence of 424 SP assignment of ipPCA, bootstrapping was performed. Admixture analysis was then performed 425 on the same data with cross-validation to determine optimal $K$. The number of $K$ ancestral 426 components was varied from $K=2$ to 100 .

427 A) ADMIXTURE result from $K=2$ and $K=55$. The latter $K$ is the minimum cross-validation error 428 reported by ADMIXTURE. The complete results of all Ks are shown in Dataset S6. SP74-94 429 contain indicine breed individuals only, and the 28 Thai individuals are assigned to SP88-90 430 (bracketed by TH). The ADMIXTURE plots show the proportion of each inferred ancestral 431 component, as indicated by different colors for each individual. The individuals were sorted 432 horizontally according to the 96 subpopulation assignments made by ipPCA. Bootstrap intervals 
433 ( $\leq 80 \%$, red circle; $80-95 \%$, yellow square; $\geq 95 \%$, green star) for each ipPCA assigned SP are 434 indicated by the symbols on the top. The plots for $\mathrm{K}=2$ and 55 are shown;

435 B) ADMIXTURE analysis of indicine individuals only (296 individuals genotyped for 44,706 436 markers) was performed varying ancestral components from $K=2$ to 30 . The plot shows the result 437 from $K=11$, which is the $K$ with minimum cross-validation error. The individuals are sorted 438 according to breed labels shown underneath the plot; the 28 Thai individuals are grouped 439 according to their assumed origins (THC, THR, THS, and THNE).

440 C) Ratio of inferred Southeast Asian ancestral component among Thai cattle. 28 ADMIXTURE 441 analyses were performed by introducing a single Thai individual to 268 indicine cattle from other 442 non-Thai breeds. The minimum cross-validation error was observed at $K=8$ in all 28 443 ADMIXTURE runs. The plot shows Q values of the inferred major Southeast Asian ancestral 444 component among the 28 Thai individuals grouped according to their assumed origins (THC, 445 THR, THS, and THNE).

446

447 Supporting information

448 Table S1. List of researchers and projects who contributed bovine tissue samples. 449

450 Table S2. Sampling information. Measured concentration of purified DNA from 28 samples of 451 Thai cattle.

452

453 Dataset S3. Complete genotypic data from 28 indigenous Thai cattle. 454

455 Table S4. Complete list of bovine individuals and their available information. The information 456 includes breed names, breed sample size, sub-species, continent, and geographic origin. 457

Table S5. Complete list of bovine individual assignment generated by ipPCA. The table consists of subpopulations (SP1-96) with breed label information of assigned individuals. The corresponding bootstrapping assignment confidence number obtained from 100 bootstrap replicates are shown. 
464 Figure S6. Cross-validation plots for identifying suitable $K$ ancestral components. The cross465 validation errors reported from ADMIXTURE for 10 cross-validation runs are shown from $K=2$ 466 up to $K=100$. A) The plot of ADMIXTURE cross-validation error at $K=2$ to $K=100$ of 1397 467 cattle. B) The plot of ADMIXTURE cross-validation error at $K=2$ to $K=30$ of 296 indicine 468 cattle. C) The combined plots of ADMIXTURE cross-validation errors from 28 ADMIXTURE 469 experiments at $K=2$ to $K=20$ from 268 indicine cattle plus one Thai individual.

470

471 Dataset S7. The raw outputs of ADMIXTURE analyses. The $\mathrm{Q}$ values inferred by

472 ADMIXTURE for each modeled $K$ are shown for each individual, which are labeled according 473 to breed information. The data are organized into three folders, each of which represents the 474 ADMIXTURE experiments described in Fig. S5.

475

476 Figure S8. Scatter plot of eigenvalues from Principal Component 1 (PC1) and Principal 477 Component 2 (PC2) applied to the NORM breed individuals. 478

Table S9. Pairwise identity-by-descent (IBD) computed by PLINK (Purcell et al., 2007) using $480 \quad 44,706$ markers for Southeast Asian cattle. 
Figure $\mathbf{1}_{\text {(on next page) }}$

Microsoft Word - peerjclean submission.doc Images of four native Thai cattle varieties Microsoft Word - peerjclean submission. doc Figure 1 Images of four native Thai cattle varieties, A) Kho-Khaolumpoon (THR), B) Kho- Isaan (THNE), C) Kho-Lan (THC), and D) KhoChon (THS) (Image courtesy of Department of Livestock Development). The assumed geographical origins of the varieties and other Asian breeds, namely Ongole Grade (ONG), Aceh (ACE), Pesisir (PES), Brebes (BRE), Bali (BALI), and Madura (MAD), are shown. 
PeerJ Reviewing Manuscript

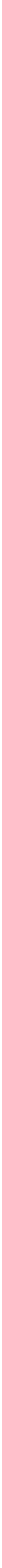


Figure 2 (on next page)

Heat map of pair-wise Fst

Microsoft Word - peerJclean submission.doc Figure 2 Heat map of pair-wise Fst. Each cell represents a group of individuals of the same breed; the heat map colors reflect Weir $\&$ Cockerham Fst values as shown by the color scale bar on the right. The data from 1397 animals genotyped for 44,706 markers were used to calculate Fst values. The gray cell represents Bison bison (OBB) and the magenta cell represents Bos javanicus (BALI), which are non-cattle reference animals. The Thai native cattle (28 individuals from THS, THR, THC, and THNE) are indicated by a green bar. Indicus, Taurine, Indicus/Taurine hybrid breeds are grouped arbitrarily, as indicated by brackets. 


\section{PeerJ Reviewing Manuscript}

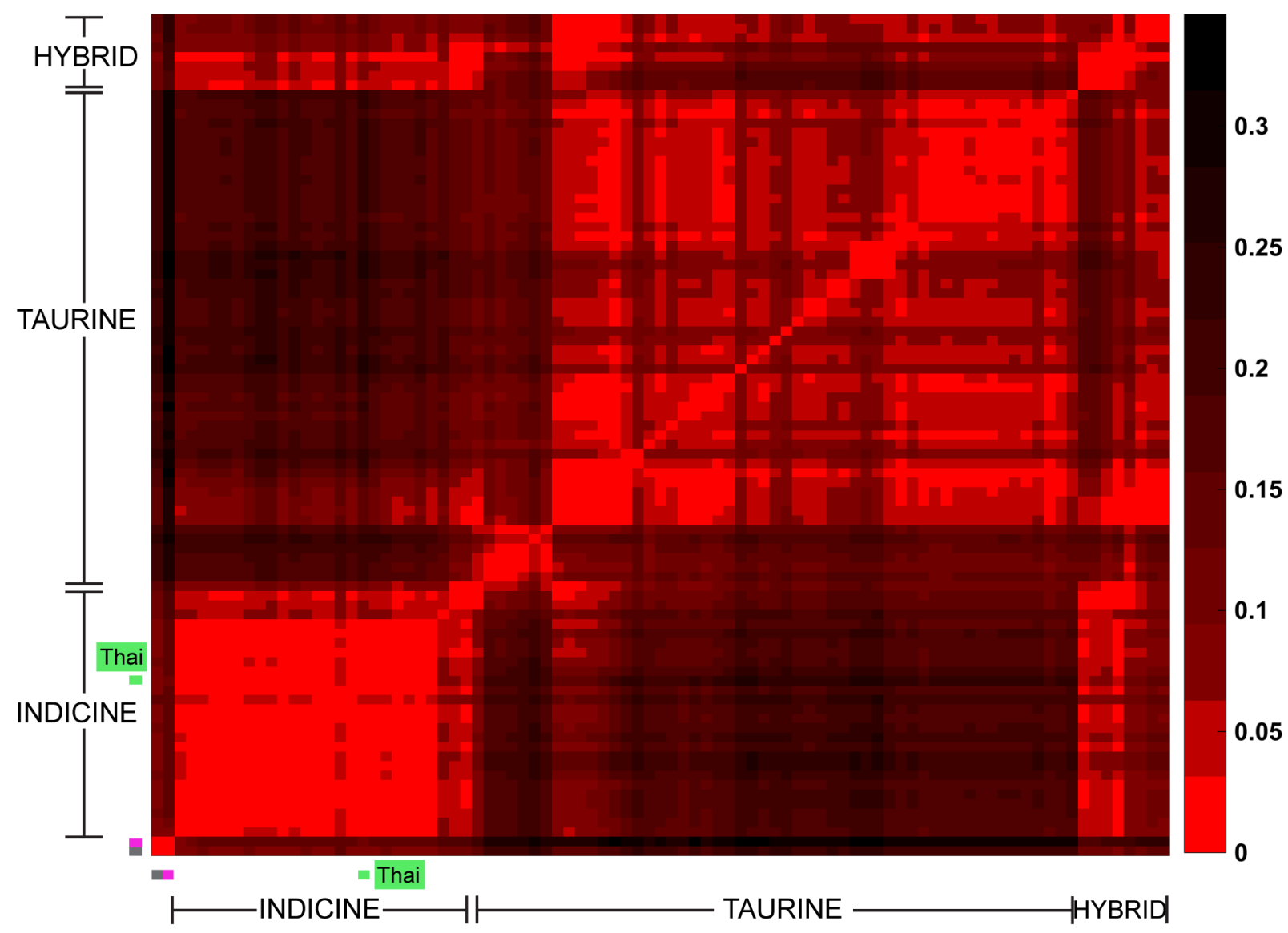




\section{Figure 3 (on next page)}

Unrooted Neighbor-Joining phylogenetic tree of cattle breeds

Microsoft Word - peerjclean submission. doc Figure 3 Unrooted Neighbor-Joining phylogenetic tree of cattle breeds. A pair-wise distance matrix between breeds was calculated based on minor allele frequency (MAF). The data from 1397 animals genotyped for 44,706 markers were used to calculate MAF values. The consensus tree is shown from 100 bootstrap replicates. The bootstrap confidence intervals ( $\leq 80 \%$, red circle; $80-95 \%$, yellow square; $\geq$ $95 \%$, green star) are indicated at each node. Taurine breeds are highlighted in blue, Hybrid breeds in green and Indicine breeds in yellow. Southeast Asian cattle are outlined in black. The Thai cattle (TH, 28 individuals from THS, THR, THC, and THNE) are highlighted in red. The non-cattle out-groups Bison bison (OBB) and Bos javanicus (BALI) are highlighted in pink. 


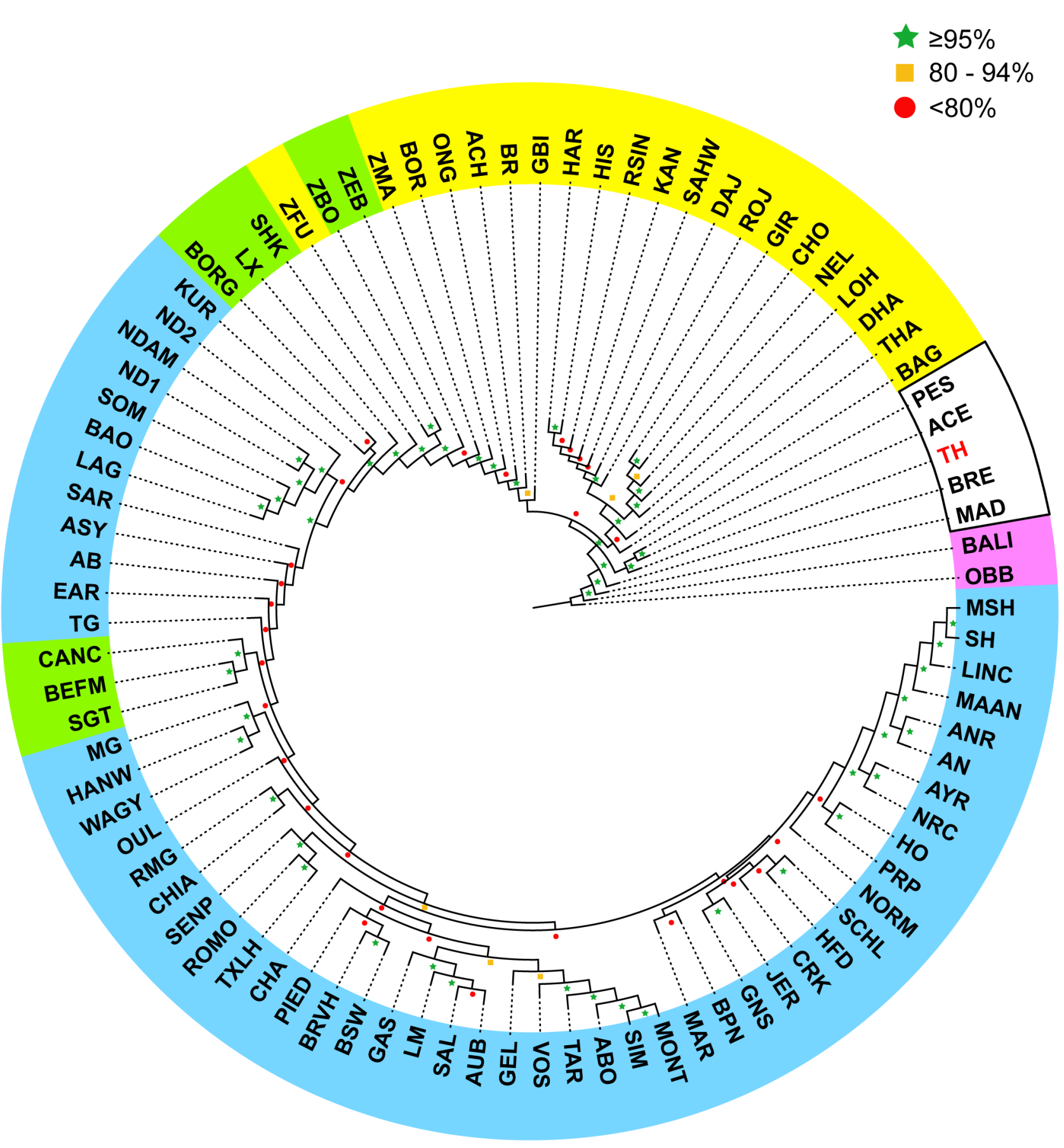




\section{Figure 4 (on next page)}

Microsoft Word - peerjclean submission.doc Combined result of ipPCA and ADMIXTURE analysis.

Microsoft Word - peerjclean submission.doc Figure 4 Combined result of ipPCA and ADMIXTURE analysis. The data from 1397 animals genotyped for 44,706 markers were used in ipPCA to assign individuals to subpopulation groups (SP1-SP96). Each SP contains individuals with no significant substructures detected by ipPCA. The SP assignment is independent of the breed label information. To assess the confidence of SP assignment of ipPCA, bootstrapping was performed. Admixture analysis was then performed on the same data with cross-validation to determine optimal $K$. The number of $K$ ancestral components was varied from $K=2$ to 100 . A) ADMIXTURE result from $K=2$ and $K=55$. The latter $K$ is the minimum cross-validation error reported by ADMIXTURE. The complete results of all Ks are shown in Dataset S6. SP74-94 contain indicine breed individuals only, and the 28 Thai individuals are assigned to SP88-90 (bracketed by TH). The ADMIXTURE plots show the proportion of each inferred ancestral component, as indicated by different colors for each individual. The individuals were sorted horizontally according to the 96 subpopulation assignments made by ipPCA. Bootstrap intervals ( $\leq 80 \%$, red circle; $80-95 \%$, yellow square; $\geq 95 \%$, green star) for each ipPCA assigned SP are indicated by the symbols on the top. The plots for $\mathrm{K}=2$ and 55 are shown; B) ADMIXTURE analysis of indicine individuals only (296 individuals genotyped for 44,706 markers) was performed varying ancestral components from $K=2$ to 30 . The plot shows the result from $K=11$, which is the $K$ with minimum crossvalidation error. The individuals are sorted according to breed labels shown underneath the plot; the 28 Thai individuals are grouped according to their assumed origins (THC, THR, THS, and THNE). C) Ratio of inferred Southeast Asian ancestral component among Thai cattle. 28 ADMIXTURE analyses were performed by introducing a single Thai individual to 268 indicine cattle from other non-Thai breeds. The minimum cross-validation error was observed at $\mathrm{K}=8$ in all 28 ADMIXTURE runs: The plot shows P values of the inferred major Southeast Asian 
ancestral component among the 28 Thai individuals grouped according to their assumed origins (THC, THR, THS, and THNE). 


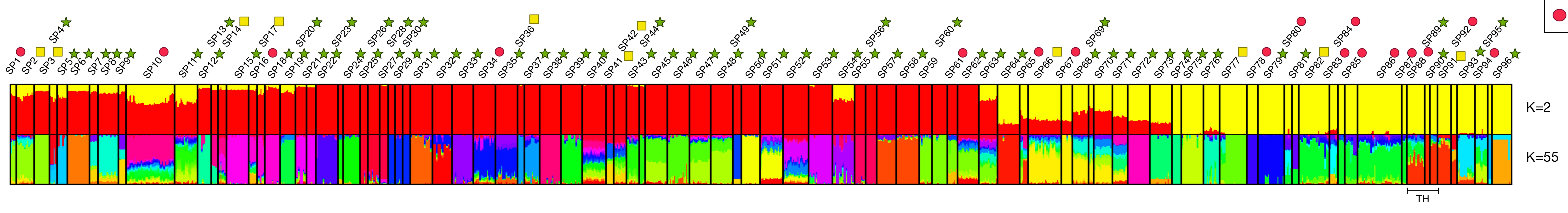

B

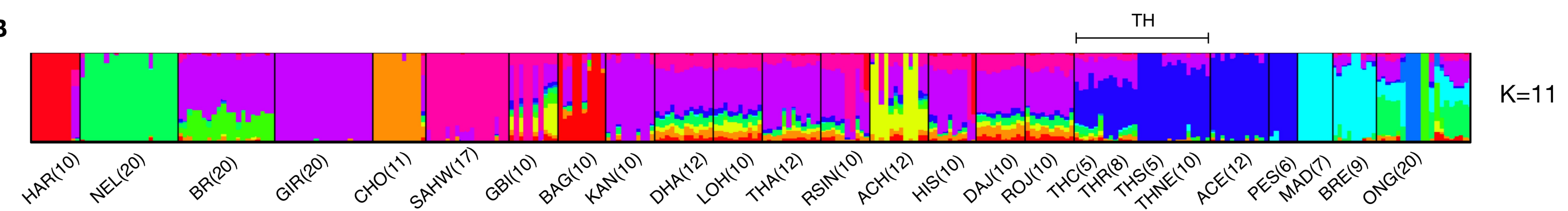

C

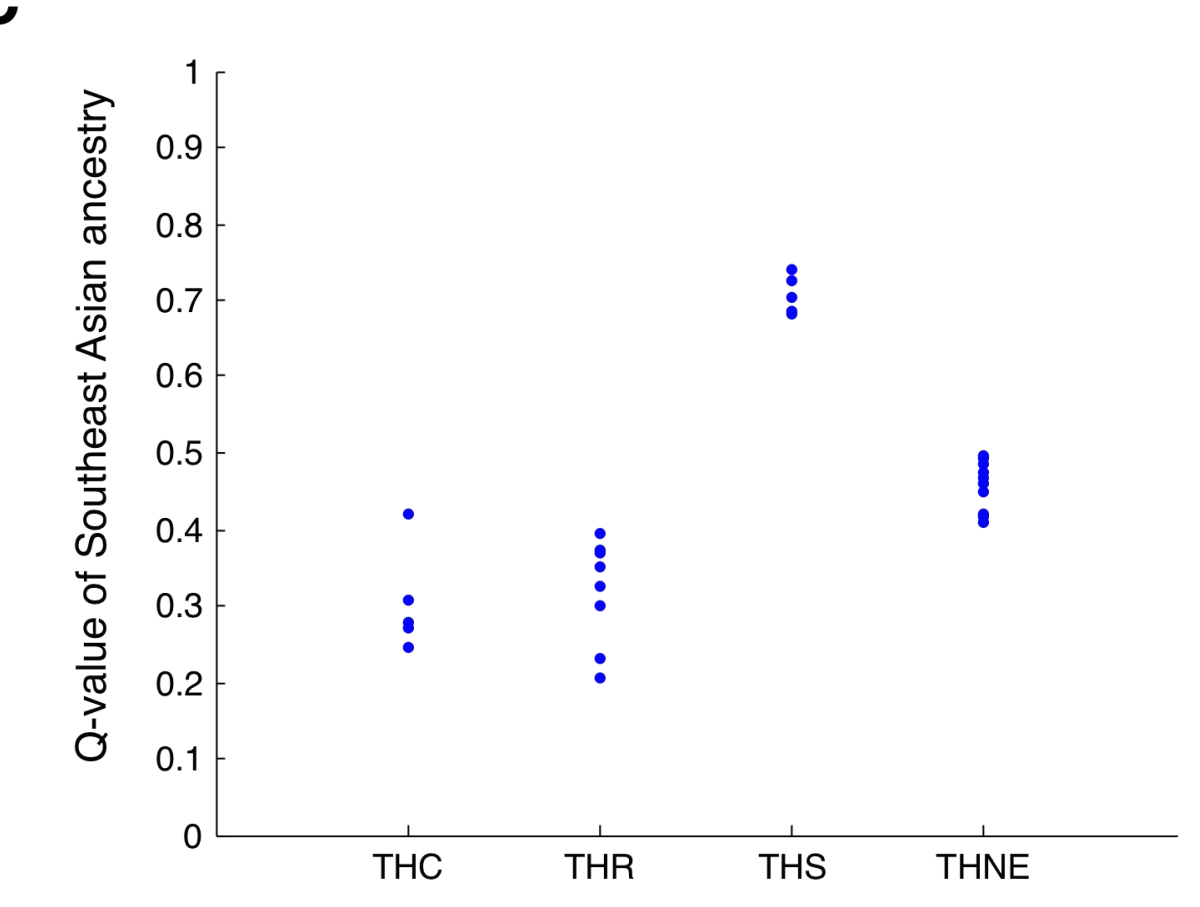

